\title{
Implementação de indicadores aplicados à gestão de suprimentos hospitalares durante a pandemia da COVID-19
}

Implementation of indicators applied to the management of hospital supplies during the COVID19 pandemic

Implementación de indicadores aplicados a la gestión de insumos hospitalarios durante la pandemia COVID-19

\author{
Marcella Viana de Souza \\ ORCID: https://orcid.org/0000-0003-2177-266X \\ Universidade Federal Fluminense, Brasil \\ E-mail: vianadsmarcella@gmail.com \\ Heloísa Sinara da Silva \\ ORCID: https://orcid.org/0000-0001-5717-7913 \\ Universidade Federal Fluminense, Brasil \\ E-mail: heloisas@id.uff.br \\ Carolina Esper Ferreira \\ ORCID: https://orcid.org/0000-0001-6029-1418 \\ Universidade Federal Fluminense, Brasil \\ E-mail: carolinaesper22@hotmail.com \\ Ranieri Carvalho Camuzi \\ ORCID: https://orcid.org/0000-0002-5584-8039 \\ Universidade Federal Fluminense, Brasil \\ E-mail: rcamuzi@id.uff.br \\ Daniele Ferreira Porto \\ ORCID: https://orcid.org/0000-0001-7536-6101 \\ Instituto Nacional de Traumatologia e Ortopedia, Brasil \\ E-mail: dporto@into.saude.gov.br
}

\begin{abstract}
Resumo
Objetivo: Analisar o uso de indicadores na aquisição de medicamentos e outros insumos de saúde e no abastecimento de estoques. Métodos: Trata-se de estudo longitudinal retrospectivo realizado em Instituto Especializado em Ortopedia e Traumatologia localizado no município do Rio de Janeiro. Os dados foram obtidos pelos próprios pesquisadores a partir de relatórios disponíveis no sistema eletrônico de gerenciamento hospitalar e planilhas internas de controle correspondentes ao período de jan/2019 a dez/2020. Resultados: Verificou-se queda no recebimento de itens entre os anos de 2019 (241) e 2020 (156), e na taxa itens homologados ao comparar 2020 [37,7 (dp=22,4)] e 2019 [58,2 $(d p=17,9)]$. A taxa de demanda não atendida apresentou 15,4\% (dp=3,2) em 2019 e 25,7\% (dp=7,1) em 2020; enquanto o tempo médio de tramitação dos processos regulares apresentou 14,5 meses $(\mathrm{dp}=1,8)$ em 2020 e 9,6 meses $(d p=2,4)$ em 2019. Conclusões: A implementação dos indicadores propiciou a verificação de deficiências na aquisição de medicamentos e outros insumos desde o período anterior à pandemia, repercutindo no desabastecimento de estoques que teve seu ápice com a COVID-19.

Palavras-chave: Administração hospitalar; Qualidade; Indicadores de gestão; Assistência farmacêutica; COVID-19.

Abstract

Objective: To analyze the use of indicators in the acquisition of medicines and other health supplies and in the supply of stocks. Methods: This is a retrospective longitudinal study carried out at the Specialized Institute in Orthopedics and Traumatology located in the city of Rio de Janeiro. The data were obtained by the researchers themselves from reports available in the hospital management system and internal control spreadsheets corresponding to the period from Jan/2019 to Dec/2020. Results: There was a drop in the receipt of items between the years 2019 (241) and 2020 (156), and in the rate approved items when comparing 2020 [37.7 (sd=22.4)] and 2019 [58.2 (sd=17.9)]. The unmet demand rate was $15.4 \%(\mathrm{sd}=3.2)$ in 2019 and $25.7 \%(\mathrm{sd}=7.1)$ in 2020; while the average processing time of regular processes was 14.5 months $(s d=1.8)$ in 2020 and 9.6 months $(s d=2.4)$ in 2019. Conclusions: The implementation of the indicators enabled the verification of deficiencies in the acquisition of medicines and other supplies since the period before the pandemic, resulting in the shortage of stocks that had its peak with COVID-19.
\end{abstract}

Keywords: Hospital administration; Quality; Management indicators; Pharmaceutical care; COVID-19. 


\begin{abstract}
Resumen
Objetivo: Analizar el uso de indicadores en la adquisición de medicamentos y otros insumos de salud y en el suministro de stock. Métodos: se trata de un estudio longitudinal, retrospectivo realizado en el Instituto Especializado de Ortopedia y Traumatología de la ciudad de Río de Janeiro/RJ/Brazil. Los datos fueron obtenidos a partir de informes disponibles en el sistema electrónico de gestión hospitalaria y hojas de cálculo de control interno, correspondientes al período de enero de 2019 a diciembre de 2020. Resultados: Hubo una caída en la recepción de ítems entre los años 2019 (241) y 2020 (156), y en la y en la tasa de ítems homologados, al comparar 2020 [37,7 (dt = $22,4)]$ y 2019 [58,2 (dt = 17,9)]. La tasa de demanda insatisfecha [GM1] fue del 15,4\% $(\mathrm{de}=3,2)$ en 2019 y del $25,7 \%$ $(\mathrm{de}=7,1)$ en 2020; mientras que el tiempo medio de tramitación de los procesos regulares fue de 14,5 meses $(\mathrm{de}=$ 1,8) en 2020 y de 9,6 meses $(\mathrm{de}=2,4)$ en 2019. Conclusiones: La implementación de los indicadores permitió constatar deficiencias en la adquisición de medicamentos y otros insumos de salud desde el período anterior a la pandemia, afectando el desabastecimiento que tuvo su pico con el COVID-19.
\end{abstract}

Palabras clave: Administración Hospitalaria; Calidad; Indicadores de gestión; Servicios farmacéuticos; COVID-19.

\title{
1. Introdução
}

A identificação de uma nova cepa de alta transmissibilidade do coronavírus, a SARS-CoV-2, observada pela primeira vez na China em dezembro de 2019, representou uma ameaça global à saúde pública. Com a instauração oficial da pandemia de Covid-19 em março de 2020 (Organização Pan-Americana de Saúde, 2020), ações de gestão reativa na saúde foram implementadas em caráter emergencial no enfrentamento ao novo coronavírus conhecido por ocasionar uma síndrome respiratória aguda grave (Silveira \& Oliveira, 2020).

Com o aumento no consumo de medicamentos atrelado às interrupções no fornecimento e na produção de tais insumos, configurou-se o risco proeminente de desabastecimento nas instituições de saúde. No âmbito do Sistema Único de Saúde (SUS), o aumento das demandas no nível assistencial exigiram dos gestores medidas estratégicas frente às instabilidades no abastecimento de estoques e o compromisso de reexaminar e readequar os processos essenciais (Silveira \& Oliveira, 2020; Ying et al., 2021). Do mesmo modo, a carência na oferta de medicamentos também atingiu o setor hospitalar privado, tendo o maior desafio no manejo da crise observado na área de suprimentos, devido à escassez e custos elevados de materiais e medicamentos no mercado (Paula et al., 2020).

Nesse sentido, a importância na reestruturação da assistência farmacêutica tem sido relatada especialmente no tocante aos serviços gerenciais de abastecimento de tecnologias na saúde, a fim de propor mecanismos de monitoramento e vigilância contínuas para detecção precoce de possível quadro de desabastecimento, de modo que haja tempo hábil para prevenir a escassez de insumos (Lula-Barros \& Damascena, 2021).

O desabastecimento de medicamentos nos mercados nacionais e internacionais configura um problema de grande dimensão na gestão farmacêutica hospitalar. Sob o ponto de vista econômico, o planejamento é prejudicado principalmente pela necessidade de aquisição de alternativas terapêuticas que, geralmente, são de custo mais elevado, ou diante da falta de concorrência no mercado tem seus preços elevados. Uma vez incorporadas na assistência, estas substituições aumentam a probabilidade de erros de medicação comprometendo a qualidade e a segurança no cuidado ao paciente. Para a instituição temse o cancelamento ou adiamento de procedimentos que denotam o resultado imediato e de maior visibilidade do desabastecimento (Reis \& Perini, 2008).

Diante dessas circunstâncias, a busca por ferramentas eficientes aplicadas à gestão farmacêutica se faz cada vez mais necessária, especialmente nos processos que impactam diretamente na realização de estratégias e metas das organizações. Neste contexto, destacam-se os indicadores de desempenho como elementos essenciais para o planejamento e controle dos processos nas organizações, proporcionando uma análise crítica do desempenho ao fornecer informações para estudo de melhorias (Pereira et al., 2012). Segundo Fernandes e colaboradores (2015), indicadores de desempenho são definidos como parâmetros quantificáveis associados às características de produtos e processos utilizados para melhorar o desempenho e a 
qualidade dos mesmos na organização (Fernandes et al, 2015).

Com o estabelecimento do cenário pandêmico na Europa, a readequação de indicadores na aquisição e no gerenciamento de estoques foi incluída em planos de contingenciamento nos serviços farmacêuticos hospitalares. A estratégia visou adaptar o funcionamento e a resposta do serviço às dificuldades observadas e à evolução da situação. Nessa perspectiva, as iniciativas pretendiam garantir que as eventuais alterações no âmbito de atividades chave e na integração de processos farmacêuticos não afetasse a qualidade da assistência prestada (Farinha \& Rijo, 2020).

Conforme Pontes e colaboradores (2008), o uso de indicadores de desempenho difunde-se como fator crítico de sucesso na gestão de hospitais; todavia, o setor de suprimentos carece de trabalhos que discutam a contribuição do emprego de tal ferramenta (Pontes et al., 2008). Deste modo, desenvolveu-se este estudo com o objetivo de analisar o uso de indicadores de desempenho nos processos de aquisição de medicamentos e outros insumos de saúde e de abastecimento de estoques na gestão do suprimento hospitalar.

\section{Metodologia}

Trata-se de um estudo de desenvolvimento experimental (CNPq, 2021) realizado em hospital especializado de traumatologia e ortopedia de referência nacional que atende exclusivamente pacientes do SUS.

A farmácia hospitalar se organiza em uma central e três satélites, localizadas na unidade de internação, no centro de terapia intensiva e no centro cirúrgico, com funcionamento integral (24 horas/dia, 7 dias/semana), com exceção da farmácia do centro cirúrgico, que funciona de segunda a sexta. O hospital conta também com uma farmácia ambulatorial, que tem o mesmo horário de funcionamento da farmácia satélite do centro cirúrgico. As unidades de dispensação realizam os pedidos de reposição de medicamentos e outros insumos ao estoque central em dias alternados, enquanto a farmácia ambulatorial solicita semanalmente à central de abastecimento farmacêutico (CAF).

Dada a repercussão entorno da escassez de medicamentos na pandemia, a chefia do serviço de farmácia hospitalar determinou a avaliação dos processos de aquisição de medicamentos e outros insumos de saúde e de abastecimento de estoques. Utilizou-se o método proposto por Martins e Marini (2010) para seleção dos indicadores seguindo ordenadamente as etapas apresentadas na Figura 1. Neste sentido, identificaram-se os objetos de mensuração ou variáveis estratégicas para o monitoramento dos processos, a disponibilidade e a facilidade de acesso aos dados, bem como o atendimento às características descritas em literatura para os indicadores, as quais incluíam, simplicidade, seletividade, especificidade, relevância, estabilidade e comparabilidade (Cipriano, 2009). 
Figura 1. Implementação de indicadores aplicados à gestão de suprimentos hospitalares durante a pandemia da COVID-19 (Martins \& Marini, 2010).

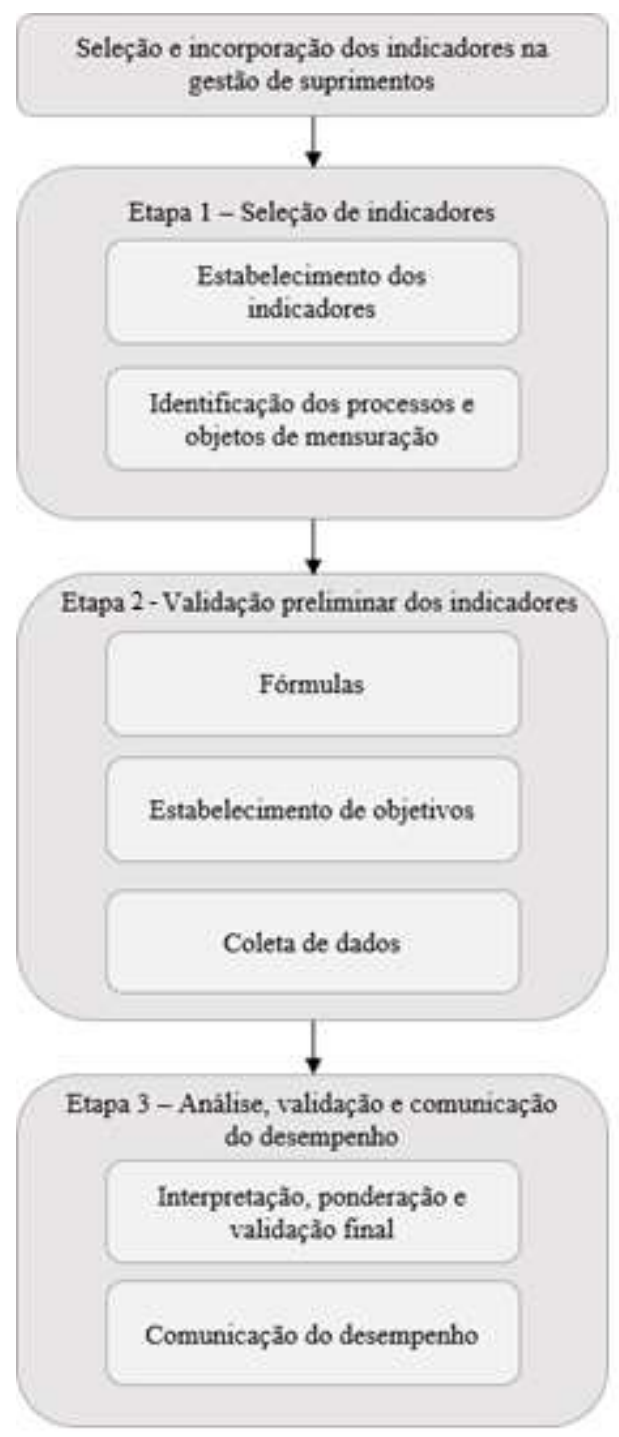

Fonte: Elaborado pelos próprios autores.

Dessa forma, as especificações dos indicadores foram definidas por meio de fórmulas e objetivos. As fórmulas foram estabelecidas para cada indicador conforme discriminadas em literatura, enquanto os objetivos foram baseados na finalidade da análise dos objetos de mensuração (Cipriano, 2009). Na coleta de dados foram definidas fontes seguras e de acesso rápido dentre aquelas disponíveis para obtenção e alimentação dos indicadores.

A validação preliminar dos indicadores selecionados consistiu na sua apresentação em reunião com a chefia da farmácia e os gestores do estoque. A partir desse momento, foi solicitada a aplicação de tais ferramentas segundo as demandas do setor.

Por fim, a validação final consistiu na análise dos resultados dos indicadores com as partes interessadas e na aprovação do uso destes para medir o desempenho e o monitoramento de riscos envolvidos nos processos pré-estabelecidos. A divulgação do desempenho foi realizada em reunião com a chefia de farmácia, gestores assistenciais e demais setores requisitantes, como a área de gerenciamento de riscos do hospital.

Para análise situacional dos processos de aquisição e abastecimento de estoques, utilizamos os dados correspondentes aos anos 2019 e 2020 para fins comparativos dos períodos anterior e durante a pandemia. A coleta destes foi realizada ao longo 
do segundo trimestre de 2021 pelos próprios pesquisadores através de relatórios disponíveis no sistema de gerenciamento hospitalar MV 2000i para as seguintes variáveis: número de itens não atendidos; itens homologados nos processos de compra; e tempo de tramitação de processos. A variável número de itens recebidos foi coletada em planilhas eletrônicas de controle de estoque.

Para o estudo foram incluídos os itens padronizados com cadastro ativo no sistema gerenciador. Foram excluídos os itens não atendidos provenientes de solicitações das enfermarias, tais como produtos multidoses e soluções parenterais de grandes volumes, bem como as solicitações da farmácia ambulatorial, uma vez que são destinadas diretamente ao CAF.

Para descrição do perfil dos indicadores e seus resultados foram calculadas a média das variáveis, bem como seu desvio padrão, exceto para o número de itens recebidos ao ano, diante da impossibilidade de relacioná-los com os meses e por se tratar de um número absoluto. O teste t de Student foi utilizado para avaliar as diferenças entre os indicadores de 2019 e 2020, adotando-se o nível de significância de 5\% (p-valor < 0,05). Além disso, empregou-se a análise de regressão (Maia, 2017) para as variáveis taxa de demanda não atendida e meses para melhor compreensão dos resultados pertencentes ao indicador. O tratamento dos dados foi conduzido no software Microsoft Excel 2010.

Uma vez que o presente estudo não utiliza dados de seres humanos na pesquisa, torna-se isento às exigências dispostas na Resolução CNS nº 510/2016 e ao trâmite no Comitê de Ética em Pesquisa.

\section{Resultados}

Os indicadores selecionados e implementados no serviço de farmácia foram: número de recebimento de itens (Consejo General de Colegios Oficiales de Farmacéuticos, 1995), taxa de demanda não atendida (Fontelene \& de Oliveira, 2015), tempo médio de tramitação dos processos regulares de compras (tempo percorrido entre a data de emissão da requisição de compra até a data da licitação) e taxa de itens homologados (Cipriano, 2009). As especificações para cada indicador objetivo e fórmula para cálculo - e as análises estatísticas são apresentadas na Tabela 1. 
Tabela 1. Indicadores de desempenho adotados durante período de pandemia de COVID-19 em Instituição de referência em Traumatologia e Ortopedia, Rio de Janeiro-BR, 2021.

\begin{tabular}{|c|c|c|c|c|c|}
\hline Indicador & Objetivo & Fórmula & 2019 & Período 2020 & p-valor ${ }^{a}$ \\
\hline $\begin{array}{l}\text { Número de } \\
\text { recebimentos de } \\
\text { itens }\end{array}$ & $\begin{array}{l}\text { Determinar } \\
\text { quantidade de } \\
\text { insumos recebidos } \\
\text { anualmente }\end{array}$ & $\begin{array}{l}\text { Número de itens } \\
\text { recebidos em } 12 \\
\text { meses }\end{array}$ & 241 & 156 & - \\
\hline $\begin{array}{c}\text { Taxa de demanda } \\
\text { não atendida }\end{array}$ & $\begin{array}{l}\text { Mensurar o } \\
\text { percentual de itens } \\
\text { não atendidos nas } \\
\text { solicitações ao } \\
\text { estoque. }\end{array}$ & $\begin{array}{l}\text { (Número de } \\
\text { solicitações não } \\
\text { atendidas/Total de } \\
\text { solicitações ao } \\
\text { estoque) x } 100^{*}\end{array}$ & $15,4 \%(3,2)$ & $25,7 \%(7,1)$ & 0,0002 \\
\hline $\begin{array}{l}\text { Tempo médio de } \\
\text { tramitação dos } \\
\text { processos } \\
\text { regulares de } \\
\text { compras }^{\mathrm{b}}\end{array}$ & $\begin{array}{l}\text { Avaliar o tempo } \\
\text { decorrido desde a } \\
\text { emissão da } \\
\text { requisição de } \\
\text { compra até a data } \\
\text { da licitação }\end{array}$ & $\begin{array}{c}\text { Tempo de } \\
\text { tramitação de } \\
\text { processos } \\
\text { licitatórios em } \\
\text { meses* }\end{array}$ & $9,6(2,4)$ & $14,5(1,8)$ & 0,0017 \\
\hline $\begin{array}{l}\text { Taxa de itens } \\
\text { homologados }\end{array}$ & $\begin{array}{l}\text { Verificar o sucesso } \\
\text { na licitação dos } \\
\text { itens. }\end{array}$ & $\begin{array}{l}\text { (Número de itens } \\
\text { homologados/total } \\
\text { de itens licitados) } \\
\text { x 100* }\end{array}$ & $58,2 \%(17,9)$ & $37,7 \%(22,4)$ & 0,0055 \\
\hline
\end{tabular}

a Teste T de student, nível de significância 5\%. ${ }^{\text {b }}$ Modalidade pregão, *Média (desvio padrão). Fonte: Dados obtidos pelos próprios autores.

Na comparação entre os períodos 2019 e 2020, foram observadas diferenças estatisticamente significantes para os indicadores, tempo médio de tramitação de processos de compra $(\mathrm{p}=0,0017)$, taxa de demanda não atendida $(\mathrm{p}=0,0002)$ e taxa de itens homologados $(\mathrm{p}=0,0055)$. Observou-se queda no recebimento de itens entre os anos de 2019 (241) e 2020 (156), enquanto o tempo médio de tramitação dos processos regulares de compras aumentou em 2020 para 14,5 meses $(\mathrm{dp}=1,8)$ quando comparado a $2019[9,6$ meses $(\mathrm{dp}=2,4)]$. A taxa de demanda não atendida apresentou aumento em torno de $10 \%$ na média anual, com taxa de 15,4\% (dp=3,2) em 2019 e 25,7\% (dp=7,1) em 2020; ao contrário da taxa de itens homologados, que apresentou redução expressiva em 2020 [37,7\% (dp=22,4)] quando comparada a 2019 [58,2\% (dp=17,9)]. As taxas de demanda não atendida são apresentadas mensalmente na Figura 2. 
Figura 2. Taxa de demanda não atendida mês a mês dos medicamentos solicitados à farmácia central nos períodos jandez/2019 e jan-dez/2020.

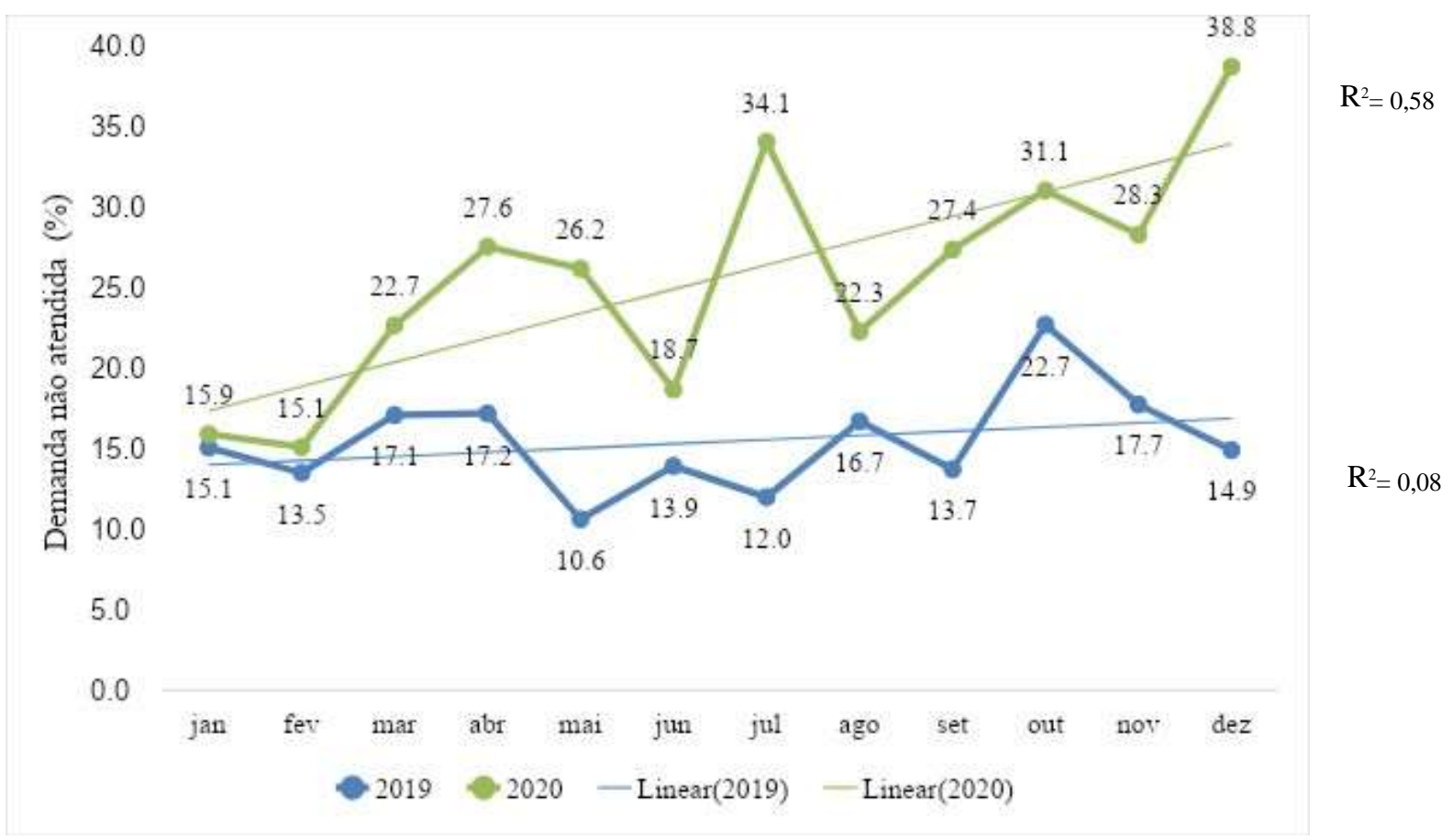

Fonte: Dados obtidos pelos próprios autores.

Ao comparar os dados dos respectivos anos, as taxas foram superiores para todos os meses em 2020, sendo os meses de julho $(34,1 \%)$ e dezembro $(38,8 \%)$ com as maiores demandas não atendidas. As menores taxas foram observadas em maio $(10,6 \%)$ e julho $(12,0 \%)$ de 2019 . Os coeficientes de determinação $\left(\mathrm{R}^{2}\right)$ foram calculados a partir da linha de tendência inserida para as variáveis taxa de demanda não atendida (y) e tempo em meses (x) em 2019 e 2020. Considerando que quando $\mathrm{R}^{2}=1$, o modelo é um perfeito ajuste para prever ou explicar um resultado, observou-se os coeficientes 0,58 (2020) e 0,08 (2019). Nesse caso, cerca de $58 \%$ da variabilidade na taxa de demanda não atendida pode ser descrita (ou explicada) pela variabilidade do passar dos meses em 2020, enquanto que 2019 apresentou quase independência linear entre as variáveis. Simultaneamente, a linha de tendência linear ascendente (2020) aponta para tendência de crescimento da demanda não atendida para os meses seguintes.

\section{Discussão}

Foram selecionados e implementados quatro indicadores de desempenho relacionados ao abastecimento de medicamentos no local de estudo. Os dados para cálculo dos indicadores foram obtidos facilmente através de relatórios administrativos do sistema de gestão institucional. A comparação entre os indicadores inferidos para os períodos de 2019 e 2020 demonstrou que há diferenças estatisticamente significantes entre todos eles, indicando que houve impacto negativo da pandemia sobre os processos de abastecimento de medicamentos e outros insumos de saúde para atendimento às demandas do local de estudo.

A aquisição de medicamentos na gestão da assistência farmacêutica é inerente às atividades prévias de programação e seleção, estando estreitamente ligada às ofertas de serviços e à cobertura assistencial dos programas de saúde (Ministério da Saúde, 2006). A farmácia hospitalar, por sua vez, ocupa importante função no contexto assistencial, visto que realiza o gerenciamento dos estoques que deve considerar, além do emprego das ferramentas de controle de consumo médio mensal, de 
estoque de segurança e ponto de ressuprimento, os prazos para realização e conclusão de processos burocráticos (Fontelene \& de Oliveira, 2015; Silva et al., 2020).

Com o aumento global no consumo de medicamentos essenciais desencadeado pela pandemia da COVID-19 em 2020, vivenciou-se no Brasil uma crise no abastecimento de medicamentos e outros insumos, dentre os quais, destacaram-se os anestésicos, sedativos, bloqueadores neuromusculares e analgésicos, que são usualmente utilizados em procedimentos cirúrgicos e denominados durante a pandemia como "kit intubação" (Dobis Bernarde \& Silva, 2021; Sociedade Brasileira de Farmácia Hospitalar (SBRAFH), 2020). Segundo autoridades governamentais, o cenário de escassez no Brasil foi atribuído a fatores como: o aumento na demanda em até oito vezes; a ausência de matérias-primas fundamentais ao processo, originárias de países que sofreram lockdown, tais como os principais China e Índia; a ocorrência de sobrepreço; e a impossibilidade de atendimento da demanda pela indústria nacional para além de um mês do consumo médio da época, mesmo aumentando a capacidade produtiva (Dobis Bernarde \& Silva, 2021).

O desabastecimento de medicamentos e o seu impacto na assistência foram sinalizados pela Sociedade Brasileira de Farmácia Hospitalar (SBRAFH) por meio de um levantamento junto a profissionais de saúde de diferentes unidades hospitalares. De acordo com o ofício, a ruptura de estoques de medicamentos e produtos para a saúde foi relatada por $87 \%$ dos profissionais participantes (SBRAFH, 2020). Da Silva e colaboradores (2021) também relataram dificuldades quanto ao abastecimento de insumos nos hospitais da cidade do Rio de Janeiro, onde os farmacêuticos tiveram que se reinventar na adoção de medidas gerenciais para contenção da COVID-19 (da Silva et al., 2021). Estes acontecimentos podem representar uma variável importante que influenciou no resultado do coeficiente de determinação (figura 2) em 2020, caracterizado pelo seu aumento comparado a 2019, dado que as significâncias das escalas de $\mathrm{R}^{2}$ dependem muito do contexto de análise e da natureza da variável dependente (y) (Maia, 2017).

No que tange ao perfil do hospital em estudo, referência em cirurgias ortopédicas eletivas, o aumento no consumo de medicamentos decorre, principalmente, do remodelamento no atendimento em 2020, dado que passou a receber pacientes de trauma de todo o estado com o intuito de aliviar outros hospitais habilitados para atendimento das demais urgências, inclusive COVID-19. De acordo com Motta Filho e colaboradores (2021), em meses de pico da pandemia - março/2020 a junho/2020 as transferências de pacientes aumentaram 124,5\% quando comparado ao mesmo período em 2019 (Motta Filho et al., 2021).

A fim de prevenir um colapso na assistência pública de saúde, priorizou-se em 2020 a abertura de processos de contingência para aquisição de medicamentos como estratégia para normalização temporária de estoques, dos quais se destacam os processos emergenciais, participações em intenção de registro de preços (IRP), atas de registro de preços e as permutas (Brasil, 1993; Dobis Bernarde \& Silva, 2021). Em decorrência disso, apesar do aumento em torno de $10 \%$ na média geral da demanda não atendida em relação ao ano anterior, houve quedas consideráveis nos meses de junho e agosto (Figura 2) influenciados pelo recebimento dos itens solicitados a partir dos processos facilitadores.

A redução no indicador número de recebimento de itens e o tempo médio de tramitação dos processos regulares de compras elevado já em 2019 são reflexos diretos das dificuldades impostas aos processos de aquisição, que podem ser justificadas por fragilidades na gestão processual intra-hospitalar perpetuadas nos últimos anos, e a organização instituída para o trâmite dos processos de compra, que possui aspectos fragmentados com compartilhamento de funções entre setores independentes e torna o percurso passível de sofrer elevação da morosidade processual. Ademais, a necessidade de adequação às novas regras processuais estabelecidas na Instrução Normativa (IN) no 40/2020 também se destaca como um fator crítico, como é possível observar pelo aumento de aproximadamente 5 meses em 2020 no tempo médio de tramitação dos processos (BRASIL, 2020). O desvio padrão elevado neste indicador (2019=2,4 meses $\sim 72$ dias; 2020=1,8 meses $\sim 54$ dias) representa 
mais um agravante para o desabastecimento, uma vez que gera inconsistências na determinação do estoque de segurança dos produtos, que, por sua vez, influenciam na programação dos quantitativos de compras.

Somam-se ao exposto, a desistência na entrega pelos fornecedores, e principalmente, a flutuação nos preços de medicamentos, que repercutiram na ruptura de estoques, sobretudo no final de 2020. A partir destes fatores é possível compreender as variações na taxa média de itens homologados, aonde verifica-se queda de aproximadamente $20 \%$ na homologação de itens licitados no período, refletindo na redução no número de itens adquiridos em 2020.

Dentre os motivos para a baixa taxa de homologação de itens, houve predominância do índice de itens desertos (sem propostas) nos processos licitatórios. Os valores das propostas acima da estimativa cotada pela administração também influenciaram diretamente no resultado deste indicador, apontando para a necessidade de otimização nas medidas de pesquisa de preços. Cabe mencionar ainda que, a falta de controle sobre a transcrição fidedigna dos dados para o formato eletrônico, bem como na alimentação das planilhas utilizadas como fontes de informação para aplicação aos indicadores pode ser considerada uma limitação deste estudo.

O conhecimento e mensuração do desempenho exerce um papel importante ao correlacionar custos e benefícios, além de avaliar o grau de satisfação dos usuários quanto à qualidade do serviço prestado. Dessa forma, a análise de resultados de indicadores tem sido uma forma simples e confiável para o monitoramento do desempenho da gestão em farmácia hospitalar (Ferreira et al., 2013). Tendo em vista a complexidade do gerenciamento de estoques, que impacta diretamente nas despesas da instituição e na manutenção da assistência, a partir deste trabalho, ratificou-se a necessidade contínua do uso de indicadores no monitoramento dos riscos envolvidos na gestão de suprimentos.

\section{Conclusão}

A implementação dos indicadores propiciou a verificação de deficiências na aquisição de medicamentos e outros insumos desde período anterior à pandemia, repercutindo no desabastecimento de estoques que teve seu ápice com a COVID19. Portanto, demonstrou-se que a utilização de indicadores contribui no acompanhamento dos processos, seus resultados e riscos de falhas, criando a oportunidade para melhores planejamentos e tomada de decisões inclusive em tempos de crise, permitindo ao farmacêutico gestor se antecipar na busca de alternativas para garantir as condições adequadas para abastecimento contínuo de insumos, evitando-se interrupções ou prejuízos na assistência.

Como sugestão para pesquisas acadêmicas futuras com enfoque neste tema, indicamos a análise paralela de indicadores de impacto direto ao paciente, utilizados como ferramentas básicas na área clínica e de cuidado ao paciente, no intuito de verificar os reflexos finais do abastecimento e de possíveis interferentes extrínsecos no ciclo assistencial.

\section{Agradecimentos}

Os autores agradecem ao apoio da equipe do serviço de farmácia hospitalar da instituição onde o estudo foi realizado.

\section{Referências}

Cipriano, S. L. (2009). Desenvolvimento de um modelo de construção e aplicação de um conjunto de indicadores de desempenho na farmácia hospitalar com foco na comparabilidade (Tese de doutorado, Universidade de São Paulo, São Paulo). https://www.teses.usp.br/teses/disponiveis/6/6135/tde-06042011161028/publico/SoniaCipriano.pdf.

Conselho Nacional de Pesquisas (CNPq). (2021). Glossário - Plataforma Lattes - CNPq. Glossário. http://lattes.cnpq.br/web/dgp/glossario?p_p_id=54_INSTANCE_QoMcDQ9EVoSc\&_54_INSTANCE_QoMcDQ9EVoSc_struts_action=\%2Fwiki_display\%2F view\&_54_INSTANCE_QoMcDQ9EVoSc_nodeName=Main\&_54_INSTANCE_QoMcDQ9EVoSc_title=Pesquisa.

Brasil. Lei No 8.666, de 21 de junho de 1993. Regulamenta o art. 37, inciso XXI, da Constituição Federal, institui normas para licitações e contratos da 
Administração Pública e dá outras providências (1993).

Consejo General de Colegios Oficiales de Farmacéuticos. (1995). Indicadores de garantía de calidad en farmacia hospitalaria. Espanha: Consejo General de Colegios Oficiales de Farmacéuticos.

da Silva, J. T., Landgraf, S. S., Sena, I. A., França, J. S., \& Silva, V. de A. (2021). Covid-19 in Rio de Janeiro/Brazil: efforts of hospital pharmacy services. Rev Bras Farm Hosp Serv Saude, 12(3), 1-8. https://doi.org/10.30968/rbfhss.2021.123.0624

Dobis Bernarde, H., \& Silva, J. F. (2021). Atuação da gestão estadual na crise dos medicamentos: um relato sobre o kit intubação [Editorial]. Acesso e cuidados especializados, 5, 252-267.

Farinha, H., \& Rijo, J. (2020). Os Farmacêuticos Hospitalares Durante a Pandemia COVID-19. Revista Portuguesa de Farmacoterapia, 12(1-2), 9-19. https://doi.org/10.25756/rpf.v12i1-2.236.

Fernandes, O., Gorman, S. K., Slavik, R. S., Semchuk, W. M., Shalansky, S., Bussières, J. F., Doucette, D., Bannerman, H., Lo,J., Shukla, S., Chan, W. W. Y., Benninger, N., MacKinnon, N. J., Bell, C. M., Slobodan, J., Lyder, C., Zed, P. J., \&amp; Toombs,K. (2015). Development of Clinical Pharmacy Key Performance Indicators for Hospital Pharmacists Using a Modified Delphi Approach. Annals of Pharmacotherapy, 49(6), 656-669. https://doi.org/10.1177/1060028015577445.

Ferreira, C. A. A., Nunes, G. ino Z., de Souza, W. I., Vianna, B. L. B., Guimarães, H. A. A., \& Azevedo, M. A. G. (2013). Monitoramento da gestão farmacêutica com o uso de indicadores em um hospital público. Revista Brasileira de Farmácia Hospitalar e Serviços de Saúde, 4(2), 14-18. https://www.rbfhss.org.br/sbrafh/article/view/158/160.

Fontelene, R. P., \& de Oliveira, T. J. C. (2015). Avaliação da etapa de aquisição para a gestão da Assistência Farmacêutica hospitalar pública. Revista Brasileira de Farmácia Hospitalar e Serviços de Saúde, 6(3), 18-22. File:///C:/Users/enfermaria/Downloads/230-Article\%20text-349-1-10-20190724.pdf.

Lula-Barros, D. S., \& Damascena, H. L. (2021). Assistência farmacêutica na pandemia da Covid-19: uma pesquisa documental. Trabalho, Educação e Saúde, 19. https://doi.org/10.1590/1981-7746-sol00323.

Martins, H. F., \& Marini, C. (2010). Um guia de governança de resultados na administração pública. Instituto Publix.

Instrução normativa $n^{\circ}$ 40, de 22 de maio de 2020, Pub. L. No. 40. Dispõe sobre a elaboração dos Estudos Técnicos Preliminares - ETP - para a aquisição de bens e a contratação de serviços e obras, no âmbito da Administração Pública federal direta, autárquica e fundacional, e sobre o Sistema ETP digital 15 (2020).

BRASIL. Ministério da Saúde. (2006). Aquisição de Medicamentos para Assistência Farmacêutica no SUS. Http://Www.Ensp.Fiocruz.Br/PortalEnsp/Judicializacao/Pdfs/284.Pdf.

Maia, A. G. (2017). Econometria: conceitos e aplicações. Saint Paul.

Motta Filho, G. da R., Leal, A. C., Amaral, M. V. G. do, Maia, P. A. V., Duarte, M. E. L., \& Bähr, G. L. (2021). Impacto das estratégias adotadas para enfrentar a pandemia de COVID-19 em um Instituto Brasileiro de referência em cirurgia de alta complexidade em Ortopedia e Traumatologia. Revista Brasileira de Ortopedia, 56(02), 161-167. Doi: https://doi.org/10.1055/s-0041-1728703.

Organização Pan-Americana de Saúde. (2020). Organização Pan-Americana de Saúde (OPAS). Https://Www.Paho.Org/Pt/Covid19.

Paula, V. R. M. de, Paula, G. M. de, Linares, F. D. C., \& Afonso, T. C. (2020). Enfrentando covid 19 em uma instituição hospitalar privada: relato de experiência. Brazilian Journal of Development, 6(11), 87727-87745. https://doi.org/10.34117/bjdv6n11-264

Pereira, L. M. V., Ungari, A. Q., \& Grande, M. M. (2012). Sistema de indicadores de desempenho para o gerenciamento de processos da farmácia de medicamentos especializados de Ribeirão Preto (SP). Revista de Administração Em Saúde, 14(56), 119-124. https://pesquisa.bvsalud.org/portal/resource/pt/lil-696236.

Reis, A. M. M, \& Perini E. (2008). Desabastecimento de medicamentos: determinantes, consequências e gerenciamento.Ciência \& Saúde Coletiva, 13, 603610. https://doi.org/10.1590/S1413-81232008000700009.

Silva, P. L., Castilho, S. R. de, \& Ferraz, C. V. V. G. (2020). Análise dos resultados da aplicação de práticas gerenciais na logística de estoque de uma farmácia hospitalar. RAHIS- Revista de Administração Hospitalar e Inovação Em Saúde, 14(2). https://doi.org/10.21450/rahis.v14i2.4088

Silveira, L. T. C. da, \& Oliveira, A. B. de. (2020). Desafios e estratégias para a organização do setor saúde frente à pandemia de COVID-19. Research, Society and Development, 9(8). https://doi.org/10.33448/rsd-v9i8.5987.

Sociedade Brasileira de Farmácia Hospitalar (SBRAF). (2020). Ofício no 037/2020, enviado ao Ministro da Saúde. Http://Www.Sbrafh.Org.Br/Inicial/WpContent/Uploads/2020/06/OFICIO-037-Ministro-Da-Saude-Oficial2.Pdf.

Pontes, A.T., Ferreira Da Silva, R., Aurélio, M., \& Pinto, C. (2008). A utilização de indicadores de desempenho no setor de suprimentos hospitalares: uma revisão de literatura. In XXVIII Encontro Nacional De Engenharia De Produção.10. Rio de Janeiro, RJ, Brasil: Associação Brasileira de Engenharia de Produção.

Ying, W., Qian, Y., \& Kun, Z. (2021). Drugs supply and pharmaceutical care management practices at a designated hospital during the COVID-19 epidemic. Research in Social and Administrative Pharmacy, 17(1), 1978-1983. https://doi.org/10.1016/j.sapharm.2020.04.001. 